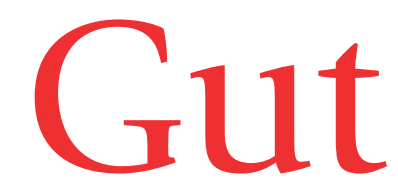

Leading article

\title{
The science, economics, and effectiveness of combination therapy for hepatitis C
}

The ideal management of hepatitis $\mathrm{C}$ infection would be to target treatment to those with progressive disease with drugs that are effective, inexpensive, and with few side effects. What is the evidence that combination antiviral therapy improves the therapeutic efficacy for this complex group of patients, and what are the implications? Hepatitis $\mathrm{C}$ virus (HCV) is a small enveloped RNA virus belonging to the family Flaviviridae. Representative population based prevalence data are not available in many countries, including the UK; the prevalence in the USA is $1.8 \% .{ }^{1}$ The majority of HCV infections in developed and developing countries are or have been caused by intravenous drug use, transfusion of unscreened blood and blood products, nosocomial transmission from inadequately sterilised instruments or unsafe injections, chronic haemodialysis, and possibly high risk sexual practices.

Infection with hepatitis $\mathrm{C}$ does not usually resolve, and $60-80 \%$ of acute infections persist. Chronic hepatitis can cause progressive fibrosis of the liver, leading to cirrhosis in $20-30 \%{ }^{2}$ Cirrhosis rarely becomes detectable before the second or third decade of infection. ${ }^{3}$ Hepatic fibrosis may occur at varying rates. However, male sex, higher alcohol use, older age at the time of infection (or perhaps index biopsy), duration of infection, and a higher necroinflammatory score at the initial liver biopsy are probable predictors of increasing fibrosis. The prediction of outcome is facilitated if fibrosis is already present. Coinfection with hepatitis B virus and/or HIV may lead to more severe disease. ${ }^{45}$ Rates of fibrosis have been estimated but the risk of eventual progression from mild disease to increasing fibrosis and eventual cirrhosis has not been adequately assessed in prospective studies. It is also unknown whether the rate of progression is linear. An increase in the incidence of hepatocellular carcinoma in the USA and Japan has been ascribed to hepatitis C infection, suggesting an expected increase in morbidity from hepatitis $\mathrm{C}$ in the next two decades. However, given the current uncertainties about the prevalence and natural history of the disease it is possible to either erroneously overestimate or underestimate the future public health consequences of hepatitis C infection. ${ }^{6}$

Considerable progress has been made in the treatment of chronic hepatitis $\mathrm{C}$, and indications for treatment must now take into account that even for patients with mild disease, improved treatment responses must be weighed against the fact that the management of patients with complications of cirrhosis is complex and costly. Therapy is recommended to reduce inflammation, fibrosis, and progression to cirrhosis or to prevent complications of cir- rhosis. Recombinant alpha interferon (IFN) monotherapy was hitherto the only licensed treatment for chronic hepatitis C. A proportion of patients show a remarkable response to treatment with rapid normalisation of alanine aminotransferase (ALT) and loss of HCV RNA in serum. However, relapse rates limited the efficacy of alpha IFN monotherapy. Given these earlier responses, the primary objective of treatment remains sustained virological response where HCV RNA remains negative by polymerase chain reaction (PCR) (albeit PCR sensitivity varies) for six months and longer after treatment has been discontinued.

For reasons that are not clear, higher response rates occur in patients with types 2 and 3 infection. ${ }^{78}$ Combination therapy with ribavirin and alpha IFN significantly enhanced sustained response rates: $49 \%$ versus $4 \%$ of patients who relapsed after treatment with alpha $2 \mathrm{~b}$ IFN alone sustained virological responses after six months of therapy with alpha IFN combined with ribavirin; and 73\% of relapsed patients infected with non-type 1 versus 30\% of those infected with type 1 responded to combination therapy. ${ }^{9}$ As a result, many patients with relapse have already been treated successfully with combination therapy.

Importantly, combination therapy has been shown to double sustained virological response rates in previously untreated patients. Large studies in the USA and Europe indicate that sustained virological response rates of $31-35 \%$ occur in patients treated with a combination of ribavirin and alpha $2 \mathrm{~b}$ IFN given for 24 weeks, and in $38-43 \%$ given for 48 weeks (compared with $13 \%$ and $19 \%$ for alpha $2 \mathrm{~b}$ alone given for 48 weeks). ${ }^{10-12}$ In patients infected with type 1 , treatment with combination ribavirin and alpha IFN for 48 rather than 24 weeks significantly improved sustained responses from $16 \%$ to $28 \%$ (whereas IFN alone resulted in sustained virological responses of only $7 \%$ ). Extending treatment beyond six months does not however significantly improve the sustained response for patients infected with non-type 1 genotypes treated with combination therapy $(69 \% v 66 \%)$. For patients with types 4,5 , and 6 , the results are intermediate $(38 \%)$ although the numbers of patients in these trials have been smaller. Combination therapy has therefore been licensed because of the greater efficacy. It should be pointed out that approximately $20 \%$ of patients discontinue treatment

Abbreviations used in this paper: $\mathrm{HCV}$, hepatitis $\mathrm{C}$ virus; IFN, interferon; ALT, alanine aminotransferase; PCR, polymerase chain reaction; IL, interleukin; PEG IFN, pegylated interferon. 
before 48 weeks, usually for insomnia, depression, irritability, or anaemia. All patients should be practising adequate contraception because of the risk of teratogenicity, and there are a number of contraindications to treatment. The side effects of combination therapy, in short, require that the treating physician is equipped to monitor and manage adverse events, and to reduce or interrupt treatment appropriately. ${ }^{13}$ Unfortunately, ribavirin accumulates in patients with renal disease, increasing haemolysis, which makes dosing difficult .

While combination therapy with ribavirin and alpha IFN is an important new therapeutic approach, it is not the final answer. Treatment is suboptimal in patients with type 1 infection and higher viral loads; a minority of these patients have a sustained virological response after 12 months of treatment. Moreover, although the distributions of patients considered for treatment varies in different regions, typically at least half to two thirds of patients have type 1 infection. There does not appear to be great benefit from combination therapy for patients who have not responded to alpha IFN monotherapy: at best, only $10-15 \%$ of patients respond to subsequent combination therapy.

A number of guidelines have been formulated to aid physicians. These include the NIH and EASL consensus statements. ${ }^{1415}$ The EASL consensus statement has the advantage of being more recent and therefore incorporates consideration of combination therapy. The EASL consensus documents asked the key questions: Which is the optimal treatment and which patients should be treated? The statement concludes that patients with moderate or severe inflammation and/or fibrosis should be treated, and that the combination of ribavirin and alpha IFN should be offered to untreated patients who are suitable for treatment. It is now difficult to justify starting treatment with alpha IFN alone. Patients with type 1 infection should be treated for 12 months, but patients with type 2 or 3 do not benefit from more than six months' treatment. It has also been suggested that for patients with type 1 and low levels of viraemia, six months' treatment may be sufficient.

Genotype should not be used as a reason to deny treatment even though patients with type 1 are less likely to respond and should be forewarned of this. The benefits of treating patients with histologically mild disease are considered to be uncertain and there is a question mark over treatment for this group. There is some disagreement on whether treatment should be discontinued in patients with type 1 infection if $\mathrm{HCV}$ RNA remains positive after three months. There are a number of special groups for whom combination antiviral treatment may be indicated for progressive disease, including liver transplant recipients, children (for whom doses of $15 \mathrm{mg} / \mathrm{kg}$ of ribavirin are being tested), and those patients who are coinfected with HIV. Increasingly, the latter patients will merit treatment if CD4 counts are maintained on antiretroviral therapy and hepatic inflammation is present. Haemophiliacs can be treated without a biopsy, for several cogent reasons, although this would appear to be a contradiction in terms. A transjugular biopsy with appropriate prophylaxis can be undertaken, particularly in older patients, if deemed clinically important. The decision to treat is made difficult in some patients, as persistently normal serum ALT may not exclude progression.

The added efficacy of ribavirin is interesting and somewhat surprising given that treatment with ribavirin alone in controlled trials had little antiviral effect. ${ }^{16}$ Ribavirin is a purine nucleoside (guanosine) analogue with immunomodulatory effects, increasing the production of type 1 cytokines (interleukin 2 (IL-2) and gamma IFN) and suppressing type 2 (IL-4) cytokines in a dose dependant manner. These shifts to a Th1 profile may be important in reducing the proinflammatory response of IFN and possibly reducing bystander damage-hence the reduction in ALT in ribavirin treated patients. These changes cannot account for all of the observed effects however. ${ }^{17}$ Analysis of the viral kinetics observed after combination therapy with ribavirin and alpha IFN suggests a synergistic action, and a critical block on viral replication and release of virions from infected cells observed in the first phase of viral decline after antiviral treatment.

Ribavirin inhibits inosine monophosphate dehydrogenase and thereby biosynthesis of guanosine triphosphate in cells, decreasing the intracellular GTP pool. However, the major antiviral effect may not be related to GTP pool depletion. Ribavirin triphosphate binds to HCV NS5b polymerase as a nucleotide substrate and is misincorporated into nascent RNA.

Other combinations for the treatment of hepatitis C seem less attractive and/or there are less data. In particular, the basis for prescribing amantadine has not been established and trials are still in progress. Novel agents, particularly new classes of drugs to inhibit the HCV polymerase, helicase, and protease are still in development. New surrogate models such as the BVDV in bovine cells, tamarins infected with GBV-B, or fetal hepatocytes differentiating into mature hepatocytes when transplanted into the peritoneal cavity of SCID mice offer new approaches to facilitate antiviral research for this disease. Glucosidase inhibitors which could block HCV replication by blocking $\mathrm{E} 1$ and E2 glycoprotein folding are being explored, ${ }^{18}$ and therapeutic vaccination is being tested in infected chimpanzees. For the present, IFN and ribavirin remain the backbone of therapy.

There is emerging evidence that pegylated IFNs (PEG IFN) may be superior to alpha IFNs. PEG IFNs are derived by covalent attachment of $43 \mathrm{kDa}$ branched polyethylene glycol moieties to IFN. This chemical change substantially prolongs the plasma half life and elimination half life by decreasing enzyme degradation and renal elimination, giving a protracted effect. The net effect is increased drug exposure, permitting once weekly dosing.

Importantly, preliminary data suggest that the altered pharmacokinetics of both PEG alpha $2 a$ and alpha $2 b$ IFNs significantly improve their antiviral effect compared with recombinant IFN. Dose ranging studies have been completed which suggest significantly superior efficacy of PEG alpha IFN compared with alpha $2 \mathrm{~b}$ and alpha $2 \mathrm{a}$ IFN, and equivalent efficacy to recombinant alpha $2 b$ IFN combined with ribavirin. Also, PEG alpha IFN alpha $2 \mathrm{a}$ $180 \mu \mathrm{g}$ weekly in patients with cirrhosis has recently been shown to cause sustained virological responses in $29 \%$ versus $6 \%$ of patients treated with alpha 2 a IFN: $53 \%$ of cirrhotic patients with non-type $1 \mathrm{HCV}$ infection responded. ${ }^{19}$ It is not yet clear if PEG IFN monotherapy will substantially improve the treatment of patients with type 1 infection and high viral loads where treatment responses are low. However, there is a reasonable expectation that the combination of PEG IFN and ribavirin will enhance responses, and these studies have begun. At least one PEG alpha IFN has been filed for licence in the European Union and will probably be available for compassionate treatment shortly.

Combination antiviral treatment strategies require funding. Funding will depend on NICE appraisal. The stated function of the National Institute for Clinical Excellence (NICE) is to appraise the clinical and cost effectiveness of new treatments in the light of guidelines. ${ }^{20}$ Combination treatment for hepatitis C (approximately $£ 5000$ per patient for six months and $£ 10000$ for one year) is not being funded by many health authorities across the UK and more funding will not be available until the NICE has issued a 
recommendation. Combination treatment for hepatitis $\mathrm{C}$ is now subject to appraisal. There is a high expectation that this recommendation will be authoritative and confer formal acceptance (or refusal) to guide health authorities to finance treatment by trusts and primary care groups.

It should be borne in mind that the evidence for clinical effectiveness of combination therapy has already been scrutinised by the European Union drug regulatory authorities and by the FDA, among others. Guidelines have been defined by the EASL consensus document-and the NIH consensus statement-after exhaustive review processes. It will be interesting to see whether the NICE appraisal contradicts or coincides with guidelines drafted in Europe. Will the NICE circumscribe the use of therapy? What independent evidence of cost effectiveness and cost benefit will be examined? Does the NICE have the resources to develop appropriate pharmoeconomic models of the treatment of hepatitis $\mathrm{C}$ with its complex subgroups? If treatment is recommended, has the NHS identified the financial resources to meet the costs of treatment?

Perhaps the important issue is the priority that the NHS is to give to treatment with combination therapy, given the uncertain and variable natural history of hepatitis C infection, and the costs of adopting treatment. The NICE and the Department of Health in the UK, and elsewhere in the world, will need to examine allocation of resources for the wider need to consider investment in treatment of hepatitis $\mathrm{C}$ at this time to reduce the future disease burden. How will the wider NHS and societal interest be best served? Can the financial burden be shared between trusts and primary care groups, and how can they work together? The Department of Health in the UK, through its agency, will be under scrutiny to make robust and wise decisions to gain the confidence of all stakeholders, including patients.

The NICE will also need to keep abreast of developments. Flexibility regarding PEG IFN may be required or the NICE may need to revise recommendations soon after they are drafted. If the enhanced efficacy of PEG IFN is substantiated and no further toxicity is noted, the indications for treatment may be extended. Perhaps patients who are currently reluctant to have treatment and who seek a cure for hepatitis $\mathrm{C}$ from alternative medicines could increasingly choose a validated form of therapy. In particular, patients who have not responded to combination therapy, including patients with cirrhosis, could be offered suppressive maintenance therapy whereby a reduction, but not elimination, of viral replication may lead to a reduction in the rate of hepatic fibrosis. There may be a particular role for PEG IFNs in this group given the convenience of once a week dosing. Suppressive therapy should lead to at least a two log decrease in HCV RNA concentrations to effect a meaningful decline in hepatic inflammatory scores. HCV RNA is probably a better guide than ALT in measuring this response in this setting. It is not clear if PEG monotherapy, rather than combination therapy, will suffice for this purpose.

There will no doubt be a premium on the cost of PEG IFN. The current high costs of combination therapy reflect the research and development costs and what the market (or particularly what the US market) will bear. Pharmaceutical companies bear the risk of drug development and are entitled to a return on their research and development costs. None the less, hopefully open market competition in the sector will drive down prices so that clinicians can provide treatment for those who need it and may benefit.

Clearly, current treatments meet only some of the criteria for optimal treatment of hepatitis C. There have, however, been real therapeutic and technological advances. In the absence of a vaccine, treatment forms part of the strategy for controlling the morbidity from hepatitis C. Hopefully the next advance in combination therapy, pegylated alpha IFN, will have twice the efficacy and half the costs of current combination therapy, making cost calculations NICE and simple.

G DUSHEIKO E BARNES G WEBSTER

Royal Free and University College School of Medicine, $S$ WHALLEY

Pond Street, Hampstead,

London NW3 2QG, UK

Correspondence to: G Dusheiko. Email: g.dusheiko@rfc.ucl.ac.uk

Conflict of interest statement. Professor Dusheiko has acted as a consultant for manufacturers of interferon, including Schering Plough, Roche and Yamanouchi/Amgen. He has received consultancy fees and honoraria to speak at symposia. His group undertakes research studies on alpha interferon and receives commercial research and development funding for this work. $\mathrm{He}$ currently holds 100 Schering Plough shares.

1 Alter MJ, Kruszon-Moran D, Nainan OV, et al. The prevalence of hepatitis $\mathrm{C}$ virus infection in the United States, 1988 through 1994. N Engl $\mathcal{F}$ Med 1999;341:556-62.

2 Dienstag JL. The natural history of chronic hepatitis $\mathrm{C}$ and what we should do about it. Gastroenterology 1997;112:651-5.

3 Poynard T, Bedossa P, Opolon P. Natural history of liver fibrosis progression in patients with chronic hepatitis C. Lancet 1997;349:825-32.

4 Cacciola I, Pollicino T, Squadrito G, Cerenzia G, Orlando ME, Raimondo G. Occult hepatitis B virus infection in patients with chronic hepatitis C G. Occult hepatitis B virus infection in pa
liver disease. N Engl f Med 1999;341:22-6.

5 Darby SC, Ewart DW, Giangrande PL, et al. Mortality from liver cancer and liver disease in haemophilic men and boys in UK given blood products contaminated with hepatitis C. Lancet 1997;350:1425-31

6 El-Serag HB, Mason AC. Rising incidence of hepatocellular carcinoma in the United States. $N$ Engl F Med 1999;340:745-50.

7 Dusheiko G, Simmonds P. Sequence variability of hepatitis $C$ virus and its clinical relevance. F Viral Hepat 1994;1:3-15.

8 Simmonds P, Mellor J, Craxi A, et al. Epidemiological, clinical and therapeutic associations of hepatitis $\mathrm{C}$ types in western European patients. f Hepatol 1996;24:517-24.

9 Davis GL. Combination therapy with interferon alfa and ribavirin as retreatment of interferon relapse in chronic hepatitis C. Semin Liver Dis 1999;19:49-55.

10 McHutchison JG, Gordon SC, Schiff ER, et al. Interferon alfa-2b alone or in combination with ribavirin as initial treatment for chronic hepatitis C. $N$ Engl f Med 1998;339:1485-92.

11 Poynard T. Interferon alfa for chronic hepatitis C infection-Reply. Lancet 1999;353:500.

12 Poynard T, Marcellin P, Lee SS, et al. Randomised trial of interferon $\alpha 2 b$ plus ribavirin for 48 weeks or for 24 weeks versus interferon $\alpha 2 \mathrm{~b}$ plus placebo for 48 weeks for treatment of chronic infection with hepatitis $\mathrm{C}$ virus. Lancet 1998;352:1426-32.

13 Dusheiko G. Side effects of alpha interferon in chronic hepatitis C. Hepatology 1997;26:112-21S.

14 National Institutes of Health consensus development conference panel statement: Management of hepatitis C. Hepatology 1997;26:2-10S.

15 EASL International Conference on Hepatitis C. Consensus statement. $\mathcal{F}$ Hepatol 1999;30:956-61.

16 Dusheiko G, Main J, Thomas H, et al. Ribavirin treatment for patients with chronic hepatitis C: Results of a placebo-controlled study. F Hepatol 1996; 25:591-8.

17 Khakoo S, Glue P, Grellier L, et al. Ribavirin and interferon alfa-2b in chronic hepatitis C: assessment of possible pharmacokinetic and pharmachronic hepatitis C: assessment of possible pharmacokinetic and

18 Zitzmann N, Mehta A, Carrouee S, et al. Imino sugars inhibit the formation and secretion of bovine diarrhea virus, a pestivirus model of hepatitis $\mathrm{C}$ and secretion of bovine diarrea virus, a pestivirus model of hepatitis $C$
virus: implications for the development of broad spectrum anti-hepatitis virus agents. Proc Natl Acad Sci USA 1999;96:11878-82.

19 Heathcote J, Shiffman M, Cooksley G, et al. Multinational evaluation of the efficacy and safety of once weekly PEGinterferon alpha2a in patients with chronic hepatitis C cirrhosis. Hepatology 1999;30:316A.

20 Rawlins M. In pursuit of quality: The National Institute for Clinical Excellence. Lancet 1999;27:1079-82. 